\title{
Interactive comment on "Temperature affects the morphology and calcification of Emiliania huxleyi strains" by Anaid Rosas-Navarro et al.
}

\section{Anonymous Referee \#2}

Received and published: 15 February 2016

Emiliania huxleyi is an important dominant species of coccolithophore. The species has been well known by its great blooming. The event has large impact on the carbon cycle of marine environment because the organism leads both photosynthesis and calcification simultaneously during this blooming event. This study attempts to evaluate the change of the production of coccolith and organic matters under variable temperatures and multiple properties through the laboratory culture experiment. The efforts will bring the useful knowledge to biogeochemists, geochemists, micropalaeontologists, oceanographers and other audience from earth science. I would like to strongly support the authors' challenge of evaluation by experimental set up.

Printer-friendly version

Questions: I would like to recommend authors to show the reason why the three strains are chosen. This species widely distributed from tropical to sub-polar region. If the Discussion paper strains originated from cooler environment were chosen, the opposite relationships 
may be shown between production and temperature. The environmental adaptation might be important to include consideration of the result. The other ecological information of them are also valuable if they are available.

P2L30ãĂĂThe authors indicate web site of Roscoff Culture Collection. It was a little bit difficult to find RCC1710 because the distribution was finished (The default setting was "distributed: Yes"). I thought some key information can be summarized in the text or table (e.g. ocean origins). It is also good option to show some details about strains by supplemental information. According to Dr Hagino, RCC 1252 was from Tsugaru straight (Japan) and RCC1710 was captured at off Nagasaki (Japan). Both places are strongly influenced by Tsushima warm current. What is the origin of IAN01? I think it is important facts to keep the reproducibility of the study. Any kind of description would be useful. Can the strain be distributed by request?

P3L12 The water samples were collected after experimental treatment?

P6L28 Why tube show positive relationship with temperature?

P7L15/P9L5 Why the malformed percentage are different among the strains?

P11L35 Control commands can be modified.

P19 Some explanation about empty places might be kindly to readers.

Interactive comment on Biogeosciences Discuss., doi:10.5194/bg-2015-591, 2016. 